\title{
Determining the metallicity of Cepheid stars in the SMC, LMC and the Galaxy
}

\author{
Marta Mottini, Francesca Primas, Martino Romaniello \\ European Southern Observatory, K. Schwarzschildstrasse 2, D-85748 \\ Garching b. München, Germany \\ Martin Groenewegen \\ Instituut voor Sterrenkunde, Celestijnenlaan 200B, B-3001 Leuven, \\ Belgium
}

\begin{abstract}
The Cepheid Period-Luminosity relation is unquestionably one of the most powerful tools at our disposal for determining the extragalactic distance scale. While significant progress has been made in the past few years towards its understanding and characterisation, both on the observational (e.g. the HST Key Project) and theoretical (e.g. non-linear pulsation models, non-LTE atmospheres etc.) sides, the debate on the influence that chemical composition may have on the PeriodLuminosity relation is still unsettled. Current estimates lead to differences in the distance as large as $15 \%$, effectively limiting the accuracy of Cepheids as distance indicators. To further tackle this problem, we have obtained high resolution spectra of a large sample of Cepheids in our Galaxy and the Magellanic Clouds. The superb quality of the data allow us to probe the detailed effects of chemical composition (alpha, iron-group, and heavy elements) over more than a factor of ten in metallicity. Here, we present the first preliminary results of the analysis of iron abundances in a sub-sample of Cepheids.
\end{abstract}

\section{Introduction}

The question if (and by how much) metallicity has any effect on the Cepheid Period-Luminosity (PL) relation is far from being settled. Recent theoretical studies give different results: some authors claim that metallicity has negligible effects on the PL relation (Baraffe \& Alibert 2001; Alibert et al. 1999; Sandage et al. 1999); others find that there is a significant dependence of the PL relation on metallicity (Bono et al. 1999; Caputo et al. 2000; Fiorentino et al. 2002).

Although most of the observational efforts aiming at deriving the chemical composition of Cepheids and its effects on the PL relation have used indirect means, like the measurement of O II in H II regions (e.g. Beaulieu et al. 1997; Sasselov et al. 1997; Kennicutt et al. 1998; Macri et al. 2001), some of them have focused on a full and direct chemical analysis (Fry \& Carney 1997; Andrievsky et al. 2002a,b; Luck et al. 2003). The goal of our project is to follow this 
second approach, i.e. to make a direct determination of the metallicity in a newly observed sample of Cepheid stars.

\section{The data sample}

The data-set has been assembled according to the following selection criteria: a large coverage of metallicities (hence, we selected stars from three different galaxies: the SMC, the LMC, and our own); the availability of well-determined intensity-mean magnitudes in the $B, V, J, H$, and $K$ bands (cf. Berdnikov \& Caldwell 2001; Groenewegen 1999; Laney \& Stobie 1992, 1994); a wide range of periods in order to cover the entire PL relation (hence we chose objects from 2 to $98 \mathrm{~d}$ in period); the availability of Hipparcos parallaxes for all Galactic Cepheids.

Our sample includes a total of 76 stars: 40 Galactic, 22 LMC, and 14 SMC Cepheids. The Magellanic Cepheids have been observed with UVES $(R=40000$, spectral coverage: $480-680 \mathrm{~nm}$ ) at the ESO-VLT and have typical $\mathrm{S} / \mathrm{N}$ of the order of 50 on the EEV CCD (i.e. for $480 \leq \lambda \leq 580 \mathrm{~nm}$ ) and $\simeq 70$ on the MIT CCD (i.e. for $580 \leq \lambda \leq 680 \mathrm{~nm}$ ). The spectra of the Galactic sample, instead, have been collected with FEROS $(R=45000$, spectral coverage: $380-880 \mathrm{~nm})$ at the ESO 1.5-m telescope in La Silla. The typical $S / N$ of these spectra is $\simeq 70$ up to $\lambda=500 \mathrm{~nm}$, but larger than 150 in the red.

\section{The analysis}

After the spectra were reduced with the help of the FEROS and UVES data reduction software pipelines, our main goal has been to develop a reliable analytical procedure in order to accurately determine the iron abundance of these stars.

First, we carefully assembled a reliable list of iron lines (both neutral and ionized). We started from a preliminary selection from the VALD database (Kupka et al. 1999; Piskunov et al. 1995) choosing lines between 450 and $650 \mathrm{~nm}$, for effective temperatures typical of Cepheids (4500-6500 K). Every line profile was then visually inspected on the observed spectra in order to detect (and eliminate) those lines affected by other elemental blends.

Secondly we measured the equivalent widths (EWs) of all the lines assembled as described above. To do this, we tested two methods: we used the Gaussian fitting option offered by the task splot in IRAF; we then developed an IDL routine (based on the line_width widget available in the FUSE IDL suite of tools) in order to speed up the process, but keeping the whole procedure interactive.

With a final list of reliable equivalent widths in hand, we then made use of the code WIDTH9 (Kurucz 1993) for the computation of our final iron abundances. For this purpose, we chose the Kurucz grid of LTE model atmospheres (Kurucz 1993). However, before being able to derive $[\mathrm{Fe} / \mathrm{H}]^{1}$ values from our measured EWs, one clearly needs to know the stellar parameters of the stars, i.e. their effective temperature, gravity, and microtubulent velocity. We derived

\footnotetext{
${ }^{1}$ where $[\mathrm{Fe} / \mathrm{H}]=\log (\mathrm{Fe} / \mathrm{H})_{*}-\log (\mathrm{Fe} / \mathrm{H})_{\odot}$
} 

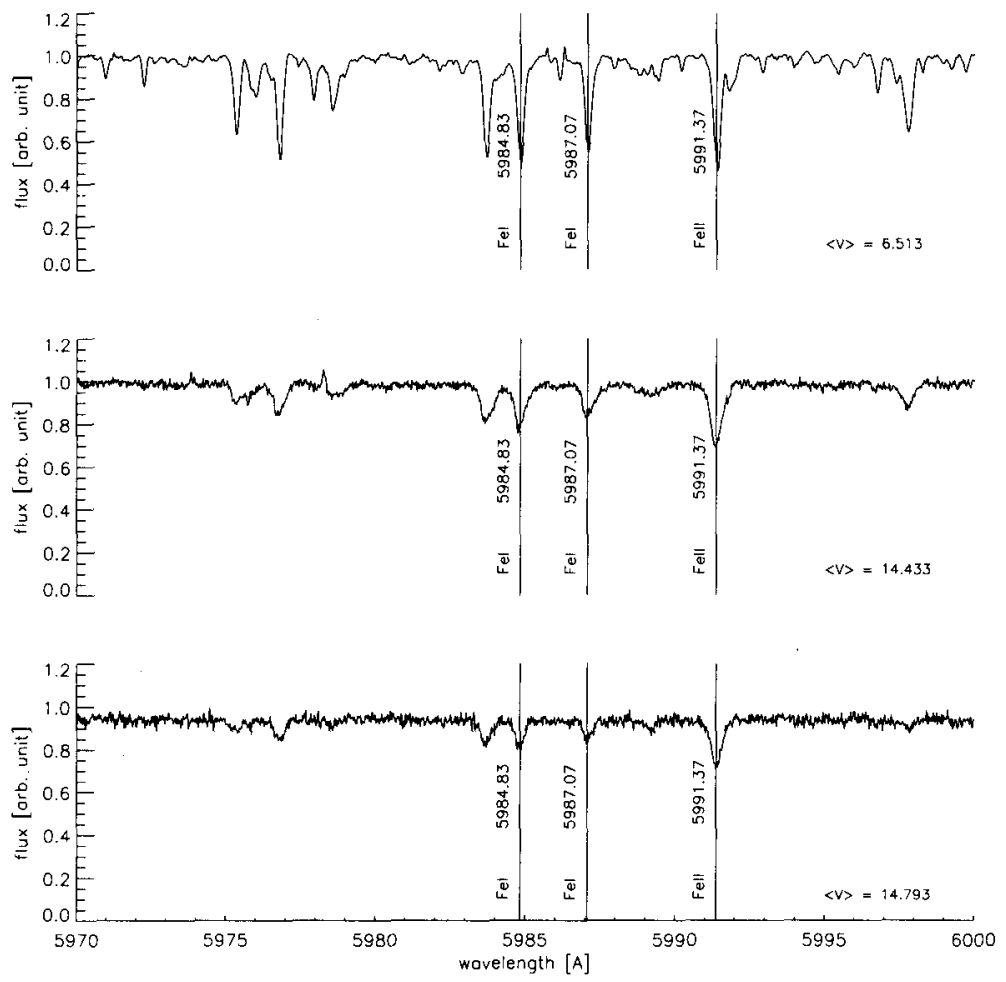

Figure 1. The same spectral range is shown for three different Cepheids of our sample, characterized by similar periods (top: Milky Way, middle: LMC, bottom: SMC).

a first guess set of stellar parameters from the photometry available in the literature (see references in Section 2) and determine spectroscopically the final set to be used in the analysis. This is an interactive process that requires running the code WIDTH9, with our final list of EWs and different model atmospheres, in order to find which model is able to minimize simultaneously the slope of $\log \epsilon(\mathrm{Fe})$ versus EW and versus the excitation potential (these constrain the microturbulence and the temperature respectively) while keeping the ionization balance (i.e. the abundance derived from FeI and from Fe II must agree) to constrain the gravity. Once such model was identified for each individual star, we were finally able to compute the iron abundance of all our stars.

\section{Preliminary results and future developments}

We have determined the iron abundances for a sub-sample of stars: 14 Galactic, 14 LMC and 12 SMC Cepheids. Fig. 1 of Romaniello et al. (these proceedings) summarises this preliminary set of results. 
Despite the confidence we have gained in the analytical steps tested so far, we still have a lot of work in front of us. In the very near future, we want to optimize our line-list (both for the number of lines to be used and for a careful check of their $g f$-values), to complete the analysis for the entire sample, and to determine iron abundances also via spectrum synthesis. A little bit more into the future, we plan to perform the full chemical analysis of the entire data-set (with special attention devoted to the $\alpha$ and to the heavy elements).

\section{References}

Alibert, Y., Baraffe, I., Hautschildt, P., Allard, F. 1999, A\&A, 344, 551

Andrievsky, S.M., Bersier, D., Kovtyukh, V.V., Luck, R.E., Maciel, W.J., Lépine, J.R.D., Beletsky, Yu.V. 2002a, A\&A, 384, 140

Andrievsky, S.M., Kovtyukh, V.V., Luck, R.E., Lépine, J.R.D., Maciel, W.J., Beletsky, Yu.V. 2002b, A\&A, 392, 491

Baraffe, I., Alibert, Y. 2001, A\&A, 371, 592

Beaulieu, J.P., Sasselov, D.D., Renault, C., Grison, P., Ferlet, R. et al. 1997, $A \& A, 318, L 47$

Berdnikov, L.N., Caldwell, J.A.R. 2001, JAD, 7, 3

Bono, G., Caputo, F., Castellani, V., Marconi, M. 1999, ApJ, 512, 711

Caputo, F., Marconi, M., Musella, I. 2000, A\&A, 354, 610

Fiorentino, G., Caputo, F., Marconi, M., Musella, I. 2002, ApJ, 576, 402

Fry, A.M., Carney, B.W. 1997, AJ, 113, 1073

Groenewegen, M.A.T. 1999, A\&ASS, 139, 245

Groenewegen, M.A.T., Oudmaijer, R.D. 2000, A\&A, 356, 849

Kennicutt, R.C., Stetson P.B., Saha, A., Kelson, D., Rawson, D.M, et al. 1998, ApJ, 498, 181

Kupka, F., Piskunov, N.E., Ryabchikova, T.A., Stempels, H.C., Weiss W.W. 1999, A\&AS, 138, 119

Kurucz, R.L. 1993, CD-ROMs \#1, 13, 18

Laney, C.D., Stobie, R.S. 1992, A\&ASS, 93, 93

Laney, C.D., Stobie, R.S. 1994, MNRAS, 266, 441

Luck, R.E., Gieren, W.P., Andrievsky, S.M., Kovtyukh, Fouqeé, P., Pont, F., Kienzle, F. 2003, A\&A, 401, 939

Macri, L.M., Bothun, G.D., Freedman, W.L., Garnavich, P.M., Jha, S., Madore, B.F., Richmond, M.W. 2001, ApJ, 559, 243

Piskunov, N.E., Kupka, F.,Ryabchikova, T.A., Weiss W.W., Jeffrey C.S. 1995, A\&AS, 112, 525

Sandage, A., Bell, R.A., Tripicco, M.J. 1999, ApJ, 522, 250

Sasselov, D.D., Beaulieu, J.P., Renault, C., Grison, P., Ferlet, R. et al. 1997, A\&A, 324, 471 